\title{
TOWARDS APPROXIMATIONS WHICH PRESERVE INTEGRALS
}

\author{
Elizabeth L Mansfield \\ Institute of Mathematics and Statistics \\ University of Kent, Canterbury \\ CT2 7NF, England \\ E.L.Mansfield@ukc.ac.uk
}

\author{
Peter E Hydon \\ Department of Mathematics and Statistics \\ University of Surrey, Guildford \\ GU2 7XH, England \\ P.Hydon@surrey.ac.uk
}

\begin{abstract}
We investigate the algorithmic approximation of ordinary differential equations having a known conservation law, with finite difference schemes which inherit a discrete version of the conservation law. We use the method of moving frames on a multispace due to Olver. We assume that the system of ODEs to be studied has a variational principle and that the conservation law arises from a variational symmetry via Noether's theorem.
\end{abstract}

\section{Categories and Subject Descriptors}

G.1.2 [Approximation]: Nonlinear approximation; I.1.2 [Symbolic and algebraic manipulation]: Algorithms; J.2 [Physical Sciences and Engineering]

\section{General Terms}

Algorithms

\section{Keywords}

moving frames, variational principles, approximation, symmetry

\section{INTRODUCTION}

It has been observed that numerical integrators which preserve energy, potential vorticity, symplecticity, integrability, and so forth, exhibit good long term approximation of physically important qualitative features. Hence substantial research effort is underway to obtain and analyse discrete approximations which inherit conservation laws or other geometric properties. We refer the reader to the excellent survey articles [1, 13] for a guide to the literature, examples and results. Approximations obtained by discretising the variational principles underlying the continuous models are of great interest [20,8]. Indeed, many physically important conserved quantities can be obtained by applying Noether's theorem to a variational principle.

Permission to make digital or hard copies of all or part of this work for personal or classroom use is granted without fee provided that copies are not made or distributed for profit or commercial advantage, and that copies bear this notice and the full citation on the first page. To copy otherwise, to republish, to post on servers or to redistribute to lists, requires prior specific permission and/or a fee.

ISSAC 2001, UWO, Canada

(C) 2001 ACM 1-58113-218-2/ 00/ 0008

$\$ 5.00$
The use of Noether's theorem to find conservation laws requires the application of symmetry analysis to differential equations and their associated Lagrangians. The computation of symmetries and conservation laws is now a standard part of computer algebra[9], requiring the use of characteristic set algorithms to simplify the overdetermined systems of differential equations that arise en route (cf. [10, 15, 18]. More recently, software packages which utilise related computations such as equivalence classes for on-line symbolic integrators of nonlinear ordinary differential equations, are being developed[3, 4, 19].

The ultimate aim of the line of investigation of the present article is to have a set of software tools which answer questions such as, "what possible numerical integration schemes exist, for the system of differential equations at hand, which possess analogues of given geometric properties?" Further, we would want to know in some detail how good they are, and how they can best be used. This is clearly a major undertaking. We begin by asking whether discretisations can be found by algorithmic methods, suited to symbolic computation, which preserve a first integral of an ordinary differential equation obtained as a conservation law of a Lagrangian. This is an important first step in the development of an algorithm to find discretisations which preserve conserved quantities of higher dimensional equations.

Suppose a given ODE is invariant under a finite dimensional Lie group $G$. The method of moving frames $[6,7]$ as it applies in the so-called multispace for curves, defined recently by Olver [17], yields a finite difference approximation which inherits the group $G$ as its symmetry group. If the ODE is an Euler-Lagrange equation and if the first integral arises via Noether's theorem from a variational symmetry, then one might hope to use a moving frame on a multipoint space to obtain an approximation which inherits the variational symmetry. Using methods similar to those recently announced in [12], there will then be a conservation law of the discretisation which relates in a natural way to the integral of the original. Here we show how this programme might work and discuss the issues that arise.

We take for our main example the Emden-Fowler ODE

$$
\Delta: \quad y^{\prime \prime}+\frac{2 y^{\prime}}{x}+y^{5}=0
$$


which is the Euler-Lagrange equation for the Lagrangian

$$
L=-x^{2}\left(y^{\prime}\right)^{2} / 2+x^{2} y^{6} / 6
$$

The group action

$$
x^{*}=x e^{2 \epsilon}, \quad y^{*}=y e^{-\epsilon}
$$

generates a variational symmetry; $L \mathrm{~d} x$ is an invariant oneform. The conservation law or first integral obtained from a straightforward application of Noether's theorem is

$$
\phi_{1}=x^{3}\left(y^{\prime}\right)^{2}+x^{2} y y^{\prime}+x^{3} y^{6} / 3 \text {. }
$$

First we discuss the multipoint space construction, and then give the moving frame for the given group action. Next we discuss an analogue, for discrete systems, of the variational methods which form the context for Noether's theorem. This is the theorem which yields conservation laws from variational symmetries. We obtain a discretisation of (1) which inherits the variational symmetry. Hence we derive a differential-difference conservation law for the difference equation, with the mesh spacing as a new independent variable. We show this conservation law has, for its continuum limit, the integral (4).

\section{MULTISPACE AND MOVING FRAMES}

One major application of multispace is that it allows differential equations and their approximations to be considered as aspects of a single mathematical construction. We give here an outline of the construction of the multispace of curves, and refer the reader to [17] for the details and proofs. We then discuss and show an example of a moving frame. The group invariants obtained from the frame will be used in the next section.

Definition 1. Let $M$ be a manifold. An $(n+1)$-pointed curve $\mathcal{C}=\left(z_{0}, \cdots z_{n} ; C\right)$ lying in $M$ consists of a smooth curve $C \subset M$ and $(n+1)$ not necessarily distinct points lying on the curve.

Definition 2. Let $\# i=\#\left\{j \mid z_{j}=z_{i}\right\}$. Two curves are equivalent, $\left(z_{0}, \cdots z_{n} ; C\right) \sim\left(\tilde{z}_{0}, \cdots \tilde{z}_{n} ; \tilde{C}\right)$ if and only if for all $i, z_{i}=\tilde{z}_{i}$ and setting $k=\# i-1$, then the $k$ th jet of $C$ at $z_{i}$ equals the $k$ th jet of $\tilde{C}$ at $z_{i}$.

Equivalence is a generalisation of the notion of $n$th order contact. If the $(n+1)$ points $z_{j}$ coincide, then the pointed curves are equivalent precisely when they have $n$th order contact. If the $(n+1)$ points are distinct, then equivalence means simply that $\tilde{z}_{i}=z_{i}$ for all $i$.

Definition 3. The multispace of curves $M^{(n)}$ on a manifold $M$ consists of the equivalence classes of the set of all $(n+1)$-pointed curves.

By construction, $M^{(n)}$ contains both $J^{(n)}(M)$, the $n$th jet bundle over $M$, as well as the set $M^{\diamond(n+1)}$, the Cartesian product of $(n+1)$ copies of $M$ minus the set of points at which any two components are equal. The intermediate cases, where some but not all the points on the curve coincide, are elements of off-diagonal Cartesian products of jet bundles.

Co-ordinates on $M^{(n)}$ are defined recursively. A typical element of $M^{(n)}$ is the equivalence class of $\left(z_{0}, \cdots z_{n} ; C\right)$ where $z=(x, f(x))$ is the curve $C$. Define $\left[z_{j}\right]_{C}=f\left(x_{j}\right)$, and

$$
\left[z_{0} z_{1} \cdots z_{k}\right]_{C}=\lim _{z \rightarrow z_{k}} \frac{\left[z_{0} \cdots z_{k-2} z\right]_{C}-\left[z_{0} \cdots z_{k-1}\right]_{C}}{x-x_{k-1}}
$$

where $z=(x, f(x)) \in C$ tends to $\left(x_{k}, f\left(x_{k}\right)\right)$ along the curve. If $z_{k} \neq z_{k-1}$ we can replace $z$ by $z_{k}$ directly in the difference quotient and ignore the limit. If all $(k+1)$ points coincide, then $\left[z_{0} \cdots z_{0}\right]_{C}=f^{(k)}\left(x_{0}\right) / k$ !. The independent variables and $k !\left[z_{0} z_{1} \cdots z_{k}\right]_{C}, k \geq 0$ form a local co-ordinate system on $M^{(n)}$.

We wish to consider conservation laws which arise from a variational symmetry, so we consider group actions on the multispace of curves. If the Lie group $G$ acts smoothly on $M$, there is an induced action on $M^{(n)}$; one simply takes the induced action on the curves and the points that lie on it. On $J^{(n)}(M)$, the action is the standard prolongation of the group action, obtainable using the chain rule of differentiation, while on $M^{\diamond(n+1)}$ the action amounts to the product action.

Definition 4. If $G \times K \rightarrow K$ is a smooth action of $G$, then a right moving frame on $K$ is a right $G$-equivariant map $\rho: K \rightarrow G$, that is, $\rho(g \cdot z)=\rho(z) g^{-1}$.

Moving frames exist if and only if the action is regular and free. In practice frames are defined, and used, only locally. We refer the reader to [6,7] for details, discussion, proofs and examples.

In practice a right moving frame is obtained by solving a set of equations of the form $\Phi(g \cdot z)=0$ for $g$ as a function of $z \in K$; that is, the frame satisfies $\Psi(\rho(z) \cdot z)=0$. One requires $\Phi$ to satisfy the conditions of the implicit function theorem so that the solution is unique. The system $\Phi=0$ is known as the set of normalisation equations.

THEOREM 1. The components of $I(z)=\rho(z) \cdot z$ are invariants.

Proof. $I(g \cdot z)=\rho(g \cdot z) g \cdot z=\rho(z) g^{-1} g \cdot z=\rho(z) \cdot z=I(z)$.

Thus, once the frame is known, the invariants are straightforward to obtain; one simply evaluates the components of $g \cdot z$ on the frame! Further, a simple replacement rule enables any invariant function to be written in terms of these invariants.

Theorem 2 (Fels-Olver-Thomas). Let $y_{1}, \ldots y_{N} b e$ local co-ordinates of a point in $z \in K$ and suppose $\rho(z) \cdot z=$ $\left(Y_{1}, \cdots Y_{N}\right)$. If $F(z)=F(g \cdot z)$ is an invariant function, then $F\left(y_{1}, \ldots y_{N}\right)=F\left(Y_{1}, \ldots Y_{N}\right)$.

Thus, to find the expression of $F$ in terms of the invariants, simply replace each $y_{i}$ with the corresponding component of $I$.

Example Consider the group action (3). The prolongation to the derivatives of $y$ is obtained by the chain rule;

$$
\frac{\mathrm{d}^{n} y^{*}}{\mathrm{~d} x^{* n}}=e^{-2 n-1} \frac{\mathrm{d}^{n} y}{\mathrm{~d} x^{n}} .
$$


To obtain the action on the coordinates of $M^{(n)}$, let $w_{k}=z_{k}^{*}$ for all $k$. Then

$$
\begin{aligned}
{\left[w_{0}\right] } & =e^{-\epsilon}\left[z_{0}\right] \\
{\left[w_{0} w_{1}\right] } & =\lim _{(x, y) \rightarrow\left(x_{0}, y_{0}\right)} \frac{e^{-\epsilon}\left(y-y_{1}\right)}{e^{2 \epsilon}\left(x-x_{1}\right)}=e^{-3 \epsilon}\left[z_{0} z_{1}\right] \\
{\left[w_{0} \cdots w_{n}\right] } & =e^{-(2 n+1) \epsilon}\left[z_{0} \cdots z_{n}\right]
\end{aligned}
$$

If we take the normalisation equation to be $\left[w_{0}\right]=1$, then the frame is given by $e^{\epsilon}=\left[z_{0}\right]$. The invariants are obtained by evaluating the $\left[w_{0} \cdots w_{k}\right]$ on the frame, whence the invariants are

$$
J_{0}=x\left[z_{0}\right]^{2}, \quad M_{k}=\left[z_{0} \cdots z_{k}\right] /\left[z_{0}\right]^{2 k+1} .
$$

The corresponding differential invariants are

$$
J_{0, \text { diff }}=x y^{2}, \quad M_{k, \text { diff }}=y^{-2 k-1} \frac{\mathrm{d}^{k} y}{\mathrm{~d} x^{k}}
$$

while the first two finite difference invariants are

$$
\begin{aligned}
M_{1, \Delta} & =\frac{1}{y_{0}^{3}} \frac{y_{1}-y_{0}}{x_{1}-x_{0}} \\
M_{2, \Delta} & =\frac{1}{y_{0}^{5}} \frac{\left(x_{1}-x_{0}\right) y_{2}+\left(x_{0}-x_{2}\right) y_{1}+\left(x_{2}-x_{1}\right) y_{0}}{\left(x_{2}-x_{0}\right)\left(x_{1}-x_{0}\right)\left(x_{2}-x_{1}\right)}
\end{aligned}
$$

where we have used the subscripts 'diff' and ' $\Delta$ ' to denote the differential and difference versions of the multispace quantities.

Looking at the Emden-Fowler equation (1), by dividing by $y^{5}$ we obtain the ODE in terms of differential invariants. Using the replacement rule, we can immediately write down a multispace approximation to the ODE which inherits the symmetry, namely

$$
M_{2}+2 M_{1} / J_{0}+1=0 .
$$

We want to somehow translate these quantities to be in terms of the discretisation variables $y_{n}$, and $n$, to obtain a discrete equation with the correct symmetry. Consider the standard mesh, $x_{n}=n h$. (This mesh is a natural choice as it is compatible with the scaling group, a point discussed below.) Since $n$ cannot vary in a continuous way, $n$ must be an invariant, and the group action induced on $h$ is then $h^{*}=$ $e^{2 \epsilon} h$. The idea of using a moving mesh was suggested by Dorodnitsyn [5]. If we translate the multi-space invariants to 'typical' discretisation co-ordinates, namely $y_{j} \rightarrow y_{n+j}$, $x_{j} \rightarrow(n+j) h$, to obtain discrete invariants, we obtain

$$
\begin{gathered}
J_{0, t}=n h y_{n}^{2}, \quad M_{1, t}=\frac{y_{n+1}-y_{n}}{h y_{n}^{3}} \\
M_{2, t}=\frac{y_{n+2}-2 y_{n+1}+y_{n}}{2 h^{2} y_{n}^{5}}
\end{gathered}
$$

where the subscript $t$ denotes 'typical' (for want of a better term). The invariants for other mesh spacings are derived similarly.

Using the standard mesh approximation in this naive way, we obtain an ordinary difference equation approximation to the Emden-Fowler equation (1). Unfortunately the resulting equation does not have an underlying discrete variational principle; it is not a discrete Euler-Lagrange equation (in the sense of [12]). Hence a conservation law cannot be obtained via Noether's theorem. We investigate how to obtain a difference equation for which there is a variational principle in the next section.

We remark that the "standard mesh" $x_{n}=n h$ is compatible with the scaling group we consider here, in the sense that scaling and discretisation using a standard mesh commute. Indeed, it has been shown that scaling groups are the only groups which commute with the usual discretisation process [2]. However, the multispace method yields an approximation for any group action. Further, just as any non-trivial group action can be transformed (in principle) to a translation group, in the so-called canonical variables, so can it be transformed to a scaling group (in the exponentiated canonical variables). Of course, the mesh compatible with the group in the original variables may be highly nonlinear. The derivation and the study of the practicality and application of compatible meshes is a topic for further study.

\section{DISCRETE VARIATIONAL METHODS AND CONSERVATION LAWS}

A rigorous analogue of the variational complex for difference equations was recently developed [12], and all proofs of our assertions appear there. We consider equations defined on one-dimensional lattices, and show the relevant calculations for our main example.

Definition 5. The shift operator acting on functions of $n$, $u_{n}$ and (a finite number of) iterates of $u_{n}$, is defined by

$$
\begin{aligned}
S\left(P_{n}\right) & =S\left(P\left(n, u_{n}, \ldots, u_{n+m}\right)\right) \\
& =P\left(n+1, u_{n+1}, \ldots, u_{n+m+1}\right) \\
& =P_{n+1} .
\end{aligned}
$$

THEOREM 3. The adjoint of the shift operator with respect to the $\ell_{2}$ inner product, is $S^{-1}$.

PROOF.

$$
\begin{aligned}
\sum S\left(P_{n}\right) Q_{n} & =\sum P_{n+1} Q_{n} \\
=\sum P_{n} Q_{n-1} & =\sum P_{n} S^{-1}\left(Q_{n}\right)
\end{aligned}
$$

by a change of dummy variable.

If a discrete Lagrangian $L_{n}$ is given, then the associated discrete Euler-Lagrange equation is

$$
E\left(L_{n}\right)=\sum_{m} S^{-m} \frac{\partial L_{n}}{\partial u_{n+m}}=0 .
$$

Definition 6. If $P_{n}=0$ is a difference equation, define its linearisation to be

$$
D_{P}(Q)=\left.\frac{\mathrm{d}}{\mathrm{d} \epsilon}\right|_{\epsilon=0} P\left(n, u_{n}+\epsilon Q_{n}, \ldots, u_{n+m}+\epsilon Q_{n+m}\right) .
$$

TheOREM 4. A difference equation $P_{n}=0$ is an EulerLagrange equation if and only if the linearisation $D_{P}$ is self adjoint with respect to the $\ell_{2}$ inner product. That is, if

$$
\sum D_{P}(Q)_{n} \cdot R_{n}=\sum Q_{n} \cdot D_{P}(R)_{n}
$$

The self-adjointness of the linearisation is the criterion which guarantees that the discretisation has a discrete variational principle. 
Returning to our example, first note that the continuous Euler-Lagrange operator acting on (2) yields $P=x^{2} \Delta$. The linearisation has the self-adjoint (Sturm-Liouville) form

$$
D_{P}(Q)=-\frac{\mathrm{d}}{\mathrm{d} x}\left(\left(-x^{2}\right) \frac{\mathrm{d}}{\mathrm{d} x} Q\right)+5 x^{2} y^{4} Q
$$

and thus we can translate this to a self-adjoint difference form quite simply, it is

$$
\left(S^{-1}-\mathrm{id}\right) f_{n}(S-\mathrm{id}) Q_{n}+g_{n} y_{n}^{4} Q_{n}=0
$$

where id is the identity operator and $f_{n}=f(n, h), g_{n}=$ $g(n, h)$ are to be determined. We do not at this point translate $x^{2}$ to a discrete quantity, as we need the freedom to ensure the necessary conditions of symmetry are met. Hence we consider the difference equation

$$
\begin{aligned}
P_{n}: & \left(S^{-1}-\mathrm{id}\right) f_{n}(S-\mathrm{id}) y_{n}+g_{n} y_{n}^{5} \\
& =-f_{n} y_{n+1}+\left(f_{n}+f_{n-1}\right) y_{n}-f_{n-1} y_{n-1}+g_{n} y_{n}^{5} \\
& =0 .
\end{aligned}
$$

Remark Recall we have taken $x_{n}=n h$, with $h$ a parameter. In general, if we regard $y_{n}, x_{n}$ as two dependent variables, then we need two equations, as there will be an Euler-Lagrange equation derived from a Lagrangian for each dependent variable.

The homotopy operator (cf. [12]) which yields the Lagrangian $L_{n}$ for an equation $P_{n}=0$ whose linearisation $D_{P}$ is self-adjoint is

$$
L_{n}=\int_{0}^{1} P_{n}(\lambda y) y_{n} \mathrm{~d} \lambda .
$$

The notation $P_{n}(\lambda y)$ means that in the expression for $P_{n}$, $y_{n+k}$ is replaced by $\lambda y_{n+k}$ for all $k$. The Lagrangian obtained from (11) is

$$
\begin{aligned}
L_{n}=-\frac{1}{2}\left(f_{n} y_{n+1} y_{n}-\right. & \left.\left(f_{n}+f_{n-1}\right) y_{n}^{2}+f_{n-1} y_{n} y_{n-1}\right) \\
& +\frac{1}{6} g_{n} y_{n}^{6} .
\end{aligned}
$$

Definition 7. A group action is a variational symmetry of a discrete Euler-Lagrange equation with Lagragian $L_{n}=$ $L_{n}\left(n, h, y_{n}\right)$ if $L_{n} \mathrm{~d} h \mathrm{~d} n$ is an invariant form.

(In [12], the notation $\Delta$ is used for $\mathrm{d} n$.)

The discretisation (11) needs to have $P=x^{2} \Delta$ (where $\Delta$ is given in (1)) for its continuum limit, while having the group action

$$
n^{*}=n, \quad h^{*}=e^{2 \epsilon} h, \quad y_{n}^{*}=e^{-\epsilon} y_{n}
$$

as a variational symmetry. This yields conditions on $f_{n}$ and $g_{n}$, the functions to be determined.

The condition $L^{*} \mathrm{~d} h^{*} \mathrm{~d} n^{*}=L \mathrm{~d} h \mathrm{~d} n$ yields

$$
\begin{aligned}
& -\frac{1}{2}\left[\left(f_{n}^{*}-f_{n}\right) y_{n+1} y_{n}+\left(f_{n}^{*}-f_{n}+f_{n-1}-f_{n-1}^{*}\right) y_{n}^{2}\right. \\
& \left.\quad+\left(f_{n-1}^{*}-f_{n-1}\right) y_{n} y_{n-1}\right]+\frac{1}{6}\left(g_{N}^{*} e^{-4 \epsilon}-g_{n}\right) y_{n}^{6}=0
\end{aligned}
$$

In this case we have

$$
f_{n}^{*}=f_{n}, \quad g_{n}^{*}=e^{4 \epsilon} g_{n}
$$

by inspection. More generally, one would obtain a set of overdetermined equations to solve.

Next, the condition that the continuum limit of $P_{n}$ is $x^{2} \Delta$ must hold. Setting $y_{n}=y(x)$, and

$$
y_{n \pm 1}=y(x \pm h)=y(x) \pm h y^{\prime}(x)+\frac{1}{2} h^{2} y^{\prime \prime}(x)+\mathcal{O}\left(h^{3}\right)
$$

we have

$$
\begin{aligned}
P_{n}= & -\frac{1}{2} h^{2} y^{\prime \prime}\left(f_{n}+f_{n-1}\right)+h y^{\prime}\left(f_{n-1}-f_{n}\right) \\
& \quad+g_{n} y^{5}+\mathcal{O}\left(h^{3}\right) \\
\sim & x^{2} y^{\prime \prime}+2 x y^{\prime}+x^{2} y^{5} \\
= & n^{2} h^{2} y^{\prime \prime}+2 n h y^{\prime}+n^{2} h^{2} y^{5}
\end{aligned}
$$

using $x=n h$. Equating coefficients we obtain

$$
f_{n}+f_{n-1}=-2 n^{2}, \quad f_{n}-f_{n-1}=-2 n, \quad g_{n}=n^{2} h^{2} .
$$

Thus

$$
f_{n}=-n(n+1), \quad g_{n}=n^{2} h^{2} .
$$

These satisfy the group action equations (15).

THEOREM 5. The equation

$$
P_{n}=n(n+1) y_{n+1}-2 n^{2} y_{n}+n(n-1) y_{n-1}+h^{2} n^{2} y_{n}^{5}=0
$$

has $P=x^{2} \Delta$ for its continuum limit, is a discrete EulerLagrange equation with Lagrangian

$$
\begin{gathered}
L_{n}=\frac{1}{2}\left(n(n+1) y_{n} y_{n+1}-2 n^{2} y_{n}^{2}+n(n-1) y_{n} y_{n-1}\right)+ \\
\frac{1}{6} h^{2} n^{2} y_{n}^{6}
\end{gathered}
$$

and has the group action (13) as a variational symmetry.

Although the homotopy operator provides a systematic technique for constructing a Lagrangian $L_{n}$, this Lagrangian is not unique. Any other function $L_{n}$ that differs from $L_{n}$ by a total difference, $(S-\mathrm{id}) H$, is also a Lagrangian for the difference equation. This freedom enables us to replace the Lagrangian (17) with the equivalent Lagrangian

$$
\begin{aligned}
\hat{L}_{n}= & L_{n}+(S-\mathrm{id})\left(\frac{1}{2} n(n-1) y_{n}\left(y_{n-1}-y_{n}\right)\right) \\
& =-\frac{1}{2} n(n+1)\left(y_{n+1}-y_{n}\right)^{2}+\frac{1}{6} h^{2} n^{2} y_{n}^{6} .
\end{aligned}
$$

Clearly, this Lagrangian tends to the continuous Lagrangian in the limit as $h \rightarrow 0$ with $x=n h$ fixed.

Finally, we construct the conservation law guaranteed by Noether's theorem. For convenience, we shall use $\hat{L}_{n}$ rather than $L_{n}$; the conservation laws of the two Lagrangians are equivalent. Taking $\mathrm{d}\left(\hat{L}_{n}\left(n, h^{*}, y_{n}^{*}\right)-e^{-2 \epsilon} \hat{L}_{n}\right) / \mathrm{d} \epsilon$ at $\epsilon=0$ yields

$$
\begin{aligned}
\left(y_{n}+2 h y_{n}^{\prime}\right) & \frac{\partial \hat{L}_{n}}{\partial y_{n}}+S\left(y_{n}+2 h y_{n}^{\prime}\right) \frac{\partial \hat{L}_{n}}{\partial y_{n+1}} \\
+ & 2 h \frac{\partial \hat{L}_{n}}{\partial h}+2 \hat{L}_{n}=0
\end{aligned}
$$


where $y_{n}^{\prime}=\partial y_{n} / \partial h$. We now mimic the use of integration by parts to obtain the conservation law (cf. [16], page 272). For the discrete part of (19), "integration by parts" takes the form

$$
S(f) g-f S^{-1} g=(S-\mathrm{id})\left(f S^{-1} g\right) .
$$

Equation (19) can then be written in the form,

$$
\begin{aligned}
& \left(y_{n}+2 h y_{n}^{\prime}\right) E\left(\hat{L}_{n}\right) \\
& +(S-\mathrm{id})\left[n(n-1)\left(y_{n}+2 h y_{n}^{\prime}\right)\left(y_{n}-y_{n-1}\right)\right] \\
& \quad+\frac{\partial}{\partial h}\left[\frac{1}{3} n^{2} h^{3} y_{n}^{6}-n(n+1) h\left(y_{n+1}-y_{n}\right)^{2}\right]=0 .
\end{aligned}
$$

The conservation law for solutions of $E\left(\hat{L}_{n}\right)=0$ is then

$$
\begin{aligned}
& (S-\mathrm{id})\left[n(n-1)\left(y_{n}+2 h y_{n}^{\prime}\right)\left(y_{n}-y_{n-1}\right)\right] \\
& \quad+\frac{\partial}{\partial h}\left[\frac{1}{3} n^{2} h^{3} y_{n}^{6}-n(n+1) h\left(y_{n+1}-y_{n}\right)^{2}\right]=0 .
\end{aligned}
$$

TheOrem 6. The continuum limit of (20) is (4).

Proof. Multiplying and dividing the first summand by $h^{2}$ and the second by $n$, setting $x=n h$ and noting $(1 / n) \mathrm{d} / \mathrm{d} h=$ $\mathrm{d} / \mathrm{d} x$, yields that the continuum limit of $(20)$ is indeed the first integral (4) of (1).

Thus the conservation law (20) is the desired discrete approximation of the integral for the discretisation (16) of the Emden-Fowler ODE (1).

In fact, $P_{n}$ is the "central difference" discretisation of $\Delta$, and the reader might well remark that we have worked very hard to find a well-known approximation. However, along the way we have obtained the discrete variational principle and the conservation law, so that we have, in fact, proved that the central difference discretisation is a "geometric integrator" for the equation.

Finally we relate the discretisation found to the multispace invariants of $\S 2$. In fact, if we had made a central difference translation of the multi-space invariants rather than the obvious 'typical' one used earlier, we would have obtained the correct answer in one go (but without realising why!) We conclude this section by conjecturing that the most important part of a second order Euler-Lagrange equation, for the purposes of finding a discrete approximation to a conservation law, will be its associated Sturm-Liouville operator, and that the requirements for the discretisation to have a self-adjoint linearisation ensure that a central difference approximation of some kind will be obtained.

\section{DISCUSSION}

Our method assumes that the ordinary differential equation being studied has a conservation law which arises via a variational symmetry. In other words, the equation is an Euler-Lagrange equation and its Lagrangian form $L \mathrm{~d} x$ is invariant under a specified group action.

The method we propose, to find a discretisation which inherits the conservation law, is as follows. First the invariants of the group action on the relevant multispace are calculated [17]. This is a "solve and back-substitute" problem. A mesh which is compatible with the group action is obtained (an open problem in general, requiring canonical co-ordinates for the group action) and the group invariants in terms of the mesh variables obtained from the multispace invariants. These mesh invariants are the building blocks for the associated discrete Lagrangian. The linearised equation is written in self-adjoint form and translated to a discretised self-adjoint equation. A Lagrangian is obtained by the homotopy operator [12], requiring symbolic integration. The undetermined coefficient functions are calculated by the need for the group action to leave invariant the Lagrangian form and to obtain the correct continuum limit, conditions which generate overdetermined systems to be solved. Finally the conservation law is obtained by the differentialdifference version of Noether's theorem. This last requires "integration by parts", code to do this for PDE systems is now well-established, along with other methods for calculating conservation laws for general PDE systems[21, 22].

We remark that first integrals can be obtained directly from general ordinary difference equations [11] even if there is no underlying Lagrangian structure.

The calculations outlined above are carried out in this article for the simple example, equation (1). Its success hints that the method should work for more general examples, but also raises many questions. If we want a robust algorithm to obtain a discretisation which inherits a conservation law, then we need to understand in more detail: (i) the method whereby a mesh which is compatible with the group action is obtained, (ii) the method whereby an operator which is self-adjoint with respect to the inner product on $L_{2}$ can be "translated" to an operator which is self-adjoint with respect to the inner product on $\ell_{2}$, such that the discrete operator has the continuous operator as a continuum limit. Our example shows that for second order Lagrangians, putting the linearised equation into Sturm-Liouville form, and using central difference co-ordinates on the multispace, are the key.

Even if these questions can be resolved in principle for wide classes of equations, the possibility of implementing our algorithm in a symbolic computing environment raises further problems. An implementation of a number of the calculations associated with moving frames for differential systems, such as the recurrence formulae for invariantised differentiation, has been implemented in Maple[14]. However, an effective implemention of the moving frame method for finding the invariants with which to write down the approximation, would require the verification of the conditions of the implicit function theorem with only algebraic tools; this is one main difficulty of the "solve and back-substitute" problem mentioned above. For particular classes of normalisation equations used to define the frame, such as polynomial equations forming a prime ideal, effective algebraic tools should exist.

Nevertheless, in practical applications, we would hope that many of the steps could be carried out symbolically.

Finally, the practicality of the "geometric" discretisations obtained needs to be investigated. Can they be implemented to yield an efficient numerical integrator, and do the numerical calculations that result give insight into physically important problems? This question is part of the wider debate on whether geometric integrators are better integrators, or not; time will tell. 
Despite the significant questions that remain, we feel our method is a potentially useful addition to the known methods for this extremely important problem, deserving of further investigation.

\section{REFERENCES}

[1] C.J. Budd and A. Iserles, Geometric integration: numerical solution of differential equations on manifolds, Phil. Trans. Royal Soc. London A $\mathbf{3 5 7}$ (1999) 945-956.

[2] C.J. Budd, B. Leimkuhler and M.D. Piggot, Scaling invariance and adaptivity. University of Bath preprint, 99/18, 1999.

http: //www . maths . bath . ac .uk/MATHEMATICS /

preprints.html

[3] E.S. Cheb-Terrab and A.D. Roche, Abel ODEs: Equivalence and Integrable Classes, Computer Physics Communications, 130 (2000) 197.

[4] E.S. Cheb-Terrab and A.D. Roche, Integrating Factors for Second Order ODEs, J. of Symbolic Computation 27 (1999) 501-519.

[5] V.A. Dorodnitsyn, Symmetry of finite-difference equations. In CRC handbook of Lie group analysis of differential equations, Vol. I. pp. 365-403. Ed. N. H. Ibragimov. CRC Press, Boca Raton. 1994.

[6] M. Fels and P.J. Olver, Moving Coframes I, Acta Appl. Math. 51 (1998) 161-213.

[7] M. Fels and P.J. Olver, Moving Coframes II, Acta Appl. Math. 55 (1999) 127-208.

[8] D. Furihata, Finite difference schemes for $\frac{\partial u}{\partial t}=\left(\frac{\partial}{\partial x}\right)^{\alpha} \frac{\delta G}{\delta u}$ that inherit energy conservation or dissipation property, J. Comp. Phys., 156 (1999) $181-205$.

[9] W. Hereman, Symbolic software for Lie symmetry analysis. In CRC Handbook of Lie Group Analysis of Differential equations, Vol III, New Trends in Theoretical Developments an Computational Methods Chapter XII. Ed. N.H. Ibragimov. CRC Press, Boca Raton. 1996.

[10] E. Hubert, Factorization-free decomposition algorithms in differential algebra J. Symb. Comp., 29 (2000) 641-662.

[11] P.E. Hydon Symmetries and first integrals of ordinary difference equations. Proc. R. Soc. Lond. A 456, (2000) 2835-28.

[12] P.E. Hydon and E.L. Mansfield, A variational complex for difference equations, preprint UKC/IMS/00/32, University of Kent, U.K., 2000.

http://www.ukc.ac.uk/ims/maths/people/

E.L.Mansfield.html

[13] R. McLachlan and G.R.W. Quispel, Six Lectures in Geometric Integration (1998).

http://www.massey.ac.nz/ RMcLachl/
[14] E.L. Mansfield, Indiff: a MAPLE package for over determined differential systems with Lie symmetry, University of Kent preprint, UKC/IMS/99/37, 1999. http://www.ukc.ac.uk/ims/maths/people/ E.L.Mansfield.html

[15] E.L. Mansfield and P.A. Clarkson, Applications of the differential algebra package diffgrob2 to classical symmetries of PDEs, J. Symb. Comp. 23 (1997) $517-533$.

[16] P.J. Olver, Applications of Lie groups to differential equations, Second Ed., Graduate Texts in Mathematics, Vol 107, Springer Verlag, New York, 1993.

[17] P.J. Olver, Geometric Foundations of Numerical Algorithms and Symmetry, University of Minnesota, 2000. http://www. math.umn.edu/ olver

[18] G.J. Reid, A.D. Wittkopf and A. Boulton, Reduction of systems of nonlinear partial differential equations to simplified involutive forms, Eur. J. Appl. Math., 7 (1996) 604-635.

[19] F. Schwarz, Solving third order differential equations with maximal symmetry group Computing 65 (2000) 155-167.

[20] J.M. Wendlandt and J.E. Marsden, Mechanical Integrators derived from a discrete variational principle, Physica D106 (1997), 223-246.

[21] T. Wolf, A comparison of four approaches to the calculation of conservation laws, Euro. J. of Appl. Math. (to appear) (1998).

http://www . maths.qmw.ac .uk/ tw/

[22] T. Wolf, A. Brand and M. Mohammadzadeh, Computer algebra algorithms and routines for the computation of conservation laws and fixing of gauge in differential expressions, J. Symb. Comp. 27 (1999) 221-238. 